\title{
Accuracy and eligibility of CBCT to digitize dental plaster casts
}

\author{
Kathrin Becker ${ }^{1,2}$, Ulf Schmücker ${ }^{3}$, Dieter Drescher ${ }^{1}$, Frank Schwarz ${ }^{2}$
}

\section{Study objectives}

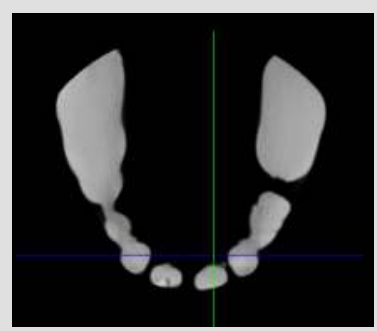

CBCT scans of plaster models

(8 devices, 10 plaster models)

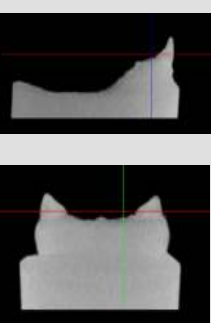

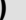

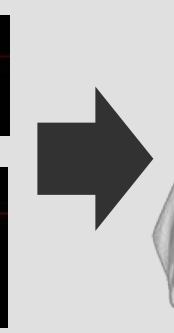

3D surface rendering

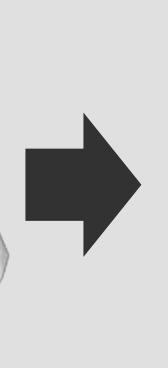

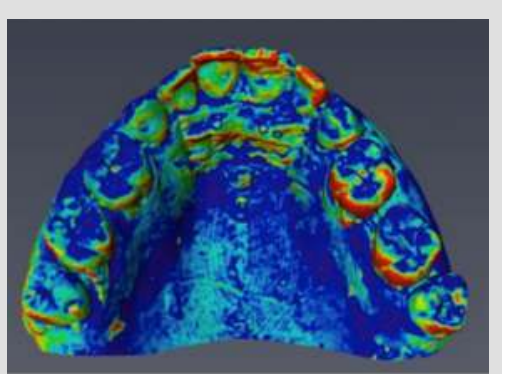

Analysis of accuracy:

Comparison with images from 5 optical devices

\section{AIMS:}

In implant dentistry, software assisted planning requires digital casts and oftentimes $\mathrm{CBCT}$ radiography. However, buying a dedicated model scanner can be expensive and might not be required. The present study aimed to assess whether digital models derived from CBCT scans and models digitized using a dedicated model scanner are of comparable accuracy.

\section{MATERIALS AND METHOD:}

A total of 20 plaster casts were digitized with eight CBCT scanners and five optical devices. Corresponding models were superimposed using six control points and subsequent iterative closest point matching. Median distances were calculated among all registered models. Boxplots were generated and the paired t-test, a Friedman test and a post-hoc Nemenyi-Test were employed for statistical comparison. Results were found significant at $p<0.05$.

\begin{tabular}{|l|c|c|c|}
\hline Device & kV & mAs & $\begin{array}{c}\text { FOV } \\
\mathbf{( c m}^{2} \mathbf{)}\end{array}$ \\
\hline Acteon, Whitefox & 120 & 8 & $8 \times 8$ \\
\hline Carestream, CS 8100 3D & 80 & 2 & $8 \times 5$ \\
\hline KaVo, OP300 3D & 90 & 6,9 & $8 \times 6$ \\
\hline KaVo 3D eXam & 120 & 37,07 & $8 \times 8$ \\
\hline Morita, Veraviewepocs 3D R100 & 80 & 3 & $8 \times 8$ \\
\hline Morita, Accuitomo & 90 & 3 & $10 \times 10$ \\
\hline $\begin{array}{l}\text { Orange Dental, Orange Green } \\
\text { 3D }\end{array}$ & 90 & 6 & $8 \times 5$ \\
\hline Planmeca, ProMax 3D Mid & 80 & 12,5 & $10 \times 10$ \\
\hline
\end{tabular}

Table 1: Scanning parameters used for the eight CBCT devices (according to manufacturer recommendations).

\section{RESULTS:}

All CBCT scanners allowed the digitization of plaster casts, but failed to reach the accuracy of the dedicated model scanners $(p<0.001)$. Median distances between CBCT and 3D scanned casts were $0.064 \pm 0.005 \mathrm{~mm}$. Qualitative differences among the CBCT scanners were detected $\left(X^{2}=85.67, p<0.001\right)$, and one CBCT scanner providing a special plaster cast scan mode was found superior to the competitors $(p<0.05)$.

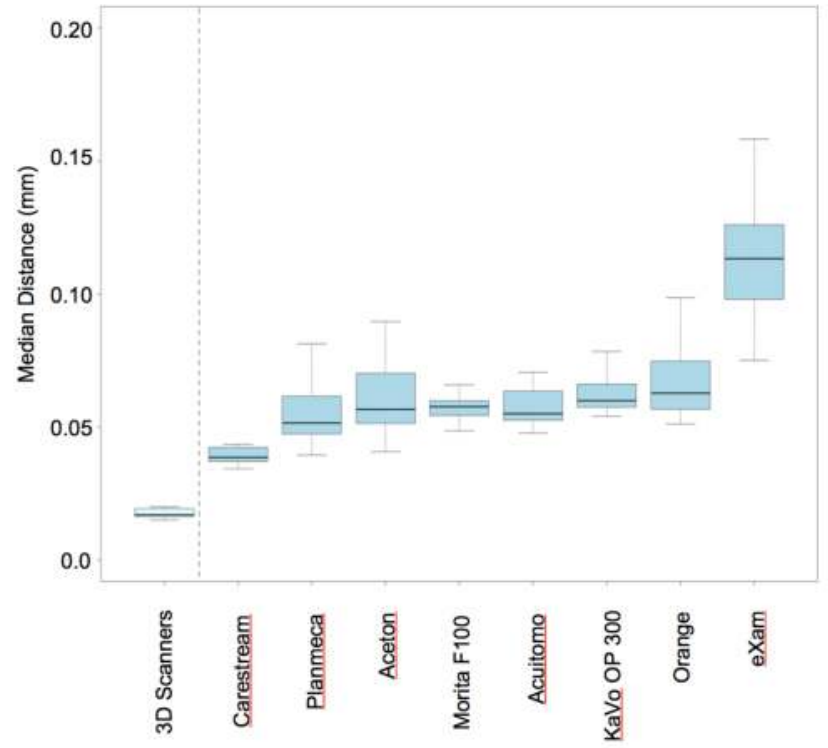

Fig 1: Boxplots presenting the distances among images derived from optical scanners (left) and between images derived from CBCT and optical digitizers (right).

\section{CONCLUSION:}

CBCT devices failed to reach the accuracy from 3D scanners, but within the limits of the study, accuracy appeared to be sufficient for digital planning and forensic purposes 